\title{
Nanoscale metal particles as nanocarriers in targeted drug delivery system
}

\begin{abstract}
In the recent years, noble metal particles such as gold $(\mathrm{Au})$ and silver $(\mathrm{Ag})$ have been used progressively as efficient and safe nanoscale drug carriers in treating malignant as cites of cancerous cells. These single crystal structures of functionalised therapeutic particles with the size less than $100 \mathrm{~nm}$ in diameter had proven offered an excellent function to modulate the oxidative stress and toxicity at affected membrane cells particularly to achieve the sitespecific delivery of drugs. This mini-review will highlight the current advances of Au and $\mathrm{Ag}$ nanoscale particles as smart chemotherapeutic molecule carriers to these malignancies in impeding cancer cell activities locally. The paper also reviewed valuable insights of their efficacy in maintaining and precisely control the drugs release level within the therapeutic windows.
\end{abstract}

Volume 4 Issue 2 - 2016

\author{
Nurul Akmal Che Lah,' Mahendran \\ Samykano,' Sonia Trigueros ${ }^{2}$ \\ 'Faculty of Mechanical Engineering, University Malaysia Pahang, \\ Malaysia \\ 2Department of Physics and Oxford Martin Programme on \\ Nanotechnology, Oxford University, UK
}

\begin{abstract}
Correspondence: Sonia Trigueros, Department of Physics and Oxford Martin Programme on Nanotechnology, Oxford University, Parks Road, OXI 3PU, UK

Email soniaTriueros@physics.ox.ac.uk
\end{abstract}

\section{Introduction}

Recent leaps in the field of oncology and therapeutic research of nano diagnostic and nano therapeutic agents has grave importance improvements in the use of nanoscale metal particles as a solid carrier for thesite-specific delivery of drugs release process. ${ }^{1-8}$ The comfort factors in using nanoscale metal particles are attained from their capability to increase the aqueous solubility of hydrophobic drugs compound, enhanced the circulation time of drugs in the blood, and repress or eliminate fast renal drugs excretion. Nevertheless, the clinical success of these agents is crucially related to the efficacy of the tiny particles to guide the chemotherapeutic drugs to the targeted cell at a designated period of time with in the body. These individual conjugates metal particles dramatically increase the cell-specific drug accumulations. ${ }^{9-12}$ and opens up the possibility of internally controlled activation (the replacement) of the delivered drug where the therapeutic effect is required in affected cell. This efficacy primarily depends on their physicochemical properties that should fit well in the context as drug conjugates. Therefore, the efficacy, in this case, is defined as maintaining the required distribution of drugs from plasma into a particular tissue or cell, there by preventing possible normal tissues and cells' damage within or close to the area being treated that might otherwise have. Nonetheless, the real challenge is a bit stiff still. The particles become thwart themselves and tend to halt the binding or producing the drug's binding incompetency at thespecific receptor, and least of these is not capable of reacting by themselves into the pathways that led down to the drug inducement system andproduced the side effect. ${ }^{13-15}$ Thus the drug delivery system demanding nanoscale particles should be exploited ina very delicate ways that can improve the capabilities of the therapeutic agent to optimise and implement the system in a safe and precise manner.

Nanosize Ag and $\mathrm{Au}$ particles have been used in elixirs and tonics for medicinal application since time immemorial due to their exciting antimicrobial properties to manipulate the fluids to generate monodisperse and uniform droplets in micro size system. ${ }^{16-18}$ The therapeutic properties of these metal particles have been identified for thousands of years. ${ }^{19,20}$ Indeed, the look on $\mathrm{Ag}$ and $\mathrm{Au}$ nanoparticles as the promising carriers become heavily researched area focus to date where the efforts in the implementation of these particles in the oncology and therapeutic fields increasing. The exponential growth in the number of well-research examples dealing with nanoscale
$\mathrm{Ag}$ and $\mathrm{Au}$ particles for diagnostic and theranostic applications comprehensively justifies their significant promises and impact of treatments' efficacy is shown in (Figure 1). This exciting discovery towards diagnostic and theranostic applications are continuously merge their way into better medical implementation not only as drug delivery 'transport', but they themselves are the drugs.

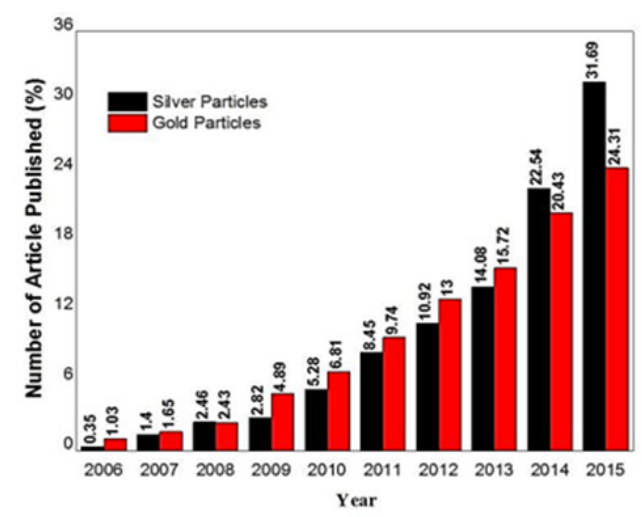

Figure I Comparison of temporal evolution in the number of scientific papers published for $\mathrm{Ag}$ and $\mathrm{Au}$ particles in drug delivery. The total number scientific papers published for $\mathrm{Ag}$ particles is lower than Au particles approximately 284 and 2423, respectively, for the past 10 years. (Source: ISI Web of Knowledge with the search terms under 'drug delivery using $\mathrm{Ag}$ and Au nanoparticles').

The assessment of the apoptotic potential of nanoscale $\mathrm{Ag}$ and $\mathrm{Au}$ particles with the diameter less than $100 \mathrm{~nm}$ have shown such great potential to facilitate site-specific drug delivery. ${ }^{21,22}$ Importantly, the diameter of the particles should not be more than $100 \mathrm{~nm}$ to avoid opsonisation and subsequent elimination of the immune system. ${ }^{23-26}$ The advantages of using $\mathrm{Ag}$ and $\mathrm{Au}$ nanoscale particles for drug delivery applicationinclude the ability of these structures to:

I. Afford enhanced drug biodistribution to specific malignancies (subcellular) sites within the body

II. Protect the therapeutic molecules from detrimental effects (i.e. molecules degradation) during transport

III. Release an effective quantity of drug through control reduction of the active therapeutic molecules ideally in or around the vicinity of the malignant target 
IV. Avoid non-specific interactions at non-targeted sites, minimising the induction of adverse reaction effects.

V. Facilitate imaging and monitoring of the treatment efficacy as the contrast agents viewed under magnetic resonance imaging (MRI).

Both nanoparticles can be guided and held in desired sites due to their unique electrical and magnetic behaviours by either magnetic or electric fields and the resultant current induced the local heating effect in cancerous regions. This local heating enhances cancerous membrane oxygenation and chemosensitivity and that trigger the release of the loaded drugs or to causes cell death by temperatureinduced apoptos is. ${ }^{27}$ Practically, the implementation of these particles as nanotherapeutic agents is observed as light scattering contrast agents monitored under MRI during photothermal ablation treatment via region-specific magnetic targeting. At the same time, their potential as effective photothermal therapy carriers facilitates their significant endorsement as sufficient absorption antibodies. However, both particles also exhibitseveral drawbacks and challenges;notably, they have greater tendency to aggregate as they encountered with larger or smaller nanoscale particle counter parts, based on the same metal content. ${ }^{28,29}$ Therefore, a combination with synthetic polymers or also known as surfactantent rapped both particles in organic stimuli-responsive matrices that form welldefined self-assemblies of polymeric nano metal structures. The layers of the adsorbed or covalently bound polymers act as an anticoagulant,preventing particlesre-aggregation. These polymeric metal particles facilitate the development of secondary functionalised metal particles attached to drugs or molecules that further protect them against recognition of immune system. These interactions are multivalent in nature, and thus the $\mathrm{Ag}$ or $\mathrm{Au}$ particles mimic the multivalent presentation of therapeutic substances on particles surface to make these functionalised nanoparticles having excellent qualities as good engineered particle carriers.

\section{Nanoscale silver particles}

In particular, nanoscale $\mathrm{Ag}$ particles are most frequently synthesised by chemical reduction method where the solutions of Ag precursor salt is chemically reduced in the presence of reducing agent. The system is further stabilised by surfactant which enhances stability (aggregation) and oxidation of the metallic Ag particles as a consequence of microscopic interaction such as the Van der Waals (between molecules and particles) and depletion forces (from excess surfactant). ${ }^{30}$ The properties of $\mathrm{Ag}$ particles for the therapeutic application does control by several factors include the morphology (size and shape), surface chemistry and surface charge as well as the state dispersion of particles. ${ }^{31,32}$

The functional properties influenced by the size and morphology of $\mathrm{Ag}$ particles mainly responsible for the de-localisation of the therapeutic drugs inside the malignancies. In most cases, particles with diameter size less than $120 \mathrm{~nm}$ were reported to be well suited for localised drug delivery applications due to the fact that they can be synthesised on a large scale with high monodispersed with almost no drug loss to the malignant sites. This point has previously been made by several authors. ${ }^{33,34}$ that the localisation behaviour of Ag particles within the malignant areas is dependent on the size as their direction can be sterically hindered in cellular matrix due to the mucoadhesives of encapsulated chemotherapeutic substances. ${ }^{35}$ The particle size of conjugate Ag particles along with their morphology does indeed give better surface chemistry effect which arises the opsonisation behaviour. The smaller the conjugate Ag particle size, the greater the accumulation inside the malignant sites that in turn shown better circulation and dispersion through different types of membrane cell. ${ }^{36}$ However, smaller particles can also increase the cytoxicity inside the membrane cells if the adherence and degradation, as well as clearance circulation, are not fully understood although $\mathrm{Ag}$ particles modulate both oxidative stress and the cellular uptake efficiently.

Interestingly, surface chemistry role during clearance or uptake in circulation accommodates the facilitation of nanotherapeutic carriers when an abundance of small nanoparticles exist within the malignancies. ${ }^{37}$ Studies revealed that nanoscale Ag particles had shown a prolonged circulation type of the half-life nanoparticles which can escape from affected cell in the tissue effectively. Long circulation is needed in chemotherapeutic treatment where the drug degradation should be negligible, therefore, undergo apoptosis where the affected cell shrink and produced condensed morphology due to the increased distribution of drug compound.

In most cases, $\mathrm{Ag}$ particles are considered as one of the photothermal the rapeuticagents due to their de-localised nature behaviour that forming a sea of the conductive electron which increased thepolarisability of these charge carriers at the surfaces ofAg particles. ${ }^{38,39}$ (Figure 2). Since Ag particles is neutrally charged particles, their cytoxicity islower than the charged ones. The attractive force between conduction electrons of $\mathrm{Ag}$ particles and malignant membrane favour the adhesion rate onto the surface of the targeted cell. Small Ag particles with diameter size less than $100 \mathrm{~nm}$ prove to alter the malignant potential effectively as well as impedes its proliferation and induces fluidity of the affected cell sites.
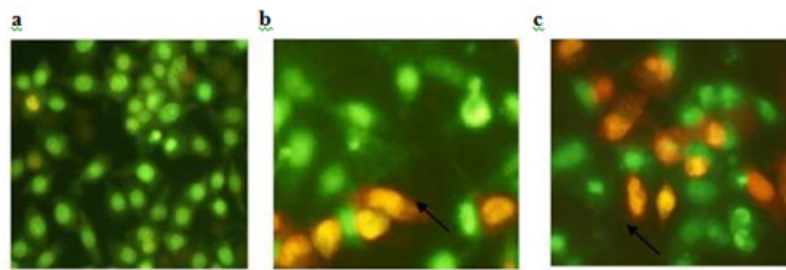

Figure 2 An example of morphological changes caused by nanoscale $\mathrm{Ag}$ particle due to the apoptosis. Ag particles treated cell exhibited condensed morphology. (Reproduced from. ${ }^{38}$ ).

\section{Nanoscale gold structures}

$\mathrm{Au}$ nanoparticles are extremely tiny solid balls made of gold with diameter vary from 5 to $100 \mathrm{~nm}^{40}$ The Au nanoparticle can be synthesized by various method such as seed-assisted growth. ${ }^{41}$ wet chemical. ${ }^{42}$ microwave assisted. ${ }^{43}$ laser ablation. ${ }^{44}$ and etc. Wet chemical is the most popular and commonly used method to synthesize $\mathrm{Au}$ nanoparticles. In this method the gold salt is reduced in the presence of reducing agent which also acts as stabilising agent. Where else, the capping reagent is used to control the growth and aggregation of the Au nanoparticles. Zeta sizer, transmission electron microscopy (TEM) and/or surface scan electron microscopy (SEM) are some of the methodologies used to characterize and determine the shape, size and size distribution of the synthesized gold nanoparticles. ${ }^{45}$

To date, a wide variety of sizes, shapes and structures of $\mathrm{Au}$ nanoparticles has been reported depending on the application at hand as shown in (Figure 3) Au nanoparticles have been shown as one of the promising and favarouble material in nanomedicine. These $\mathrm{Au}$ nanoparticles have been extensively studied and used as therapeutic agents (drug delivery). ${ }^{46}$ diagnosis agents..$^{47}$ photothermaltherapy. ${ }^{48}$ and imaging agents. ${ }^{49}$ Their tiny size, which meets the dimension of the most biological compounds, high surface area, relatively ease preparation, ease surface functionization make them particularly fascinating towards medical application. However, the interaction of $\mathrm{Au}$ nanoparticles with the surrounding biological environment 
has a significant impact towards their biological activity, as such a methodical understanding of the behaviour and nature of the interactions is essential for proper designing of these nanoparticles for diagnostics and therapeutic applications. ${ }^{50}$

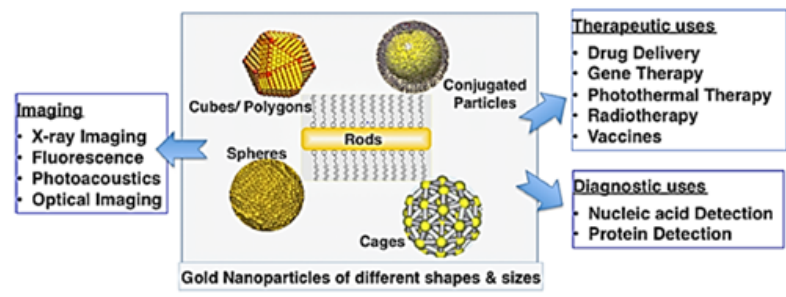

Figure 3 Wide range of potential biomedical application of Au nanoparticles depending on their synthesised size and shape (Reproduced from. ${ }^{40}$ ).

There are four main physical and chemical properties to which the $\mathrm{Au}$ nanoparticles have been tested shown to be promising candidate for clinical studies and biomedical applications: chemical inertness; surface properties; electronic structure; and optical properties. The high surface area and the relatively small number of ligands coated on smaller diameter particles cause reduced flocculation, where else the larger nanoparticles form insoluble aggregates. Additionally, the chemical inertness of this particle, facilitates gaining them in the wide range of shapes without compromising the high stability, low toxicity and immonugeneity which are essential for biomedical applications. ${ }^{51}$ Recall, most of the practice employed Au nanoparticles (Figure 4a$4 \mathrm{c})$ have been put as the preferable particle structure as drug delivery vactors in theragnostic field.

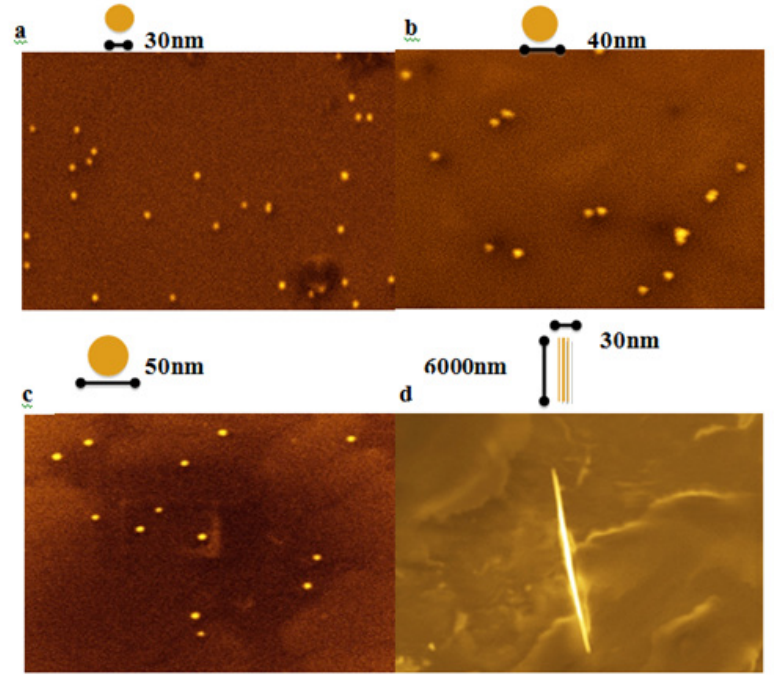

Figure 4 SEM images of Au nanoparticles (a to c) and Au nano wires (d) obtained by seed-assistance and wet chemical methods. (Images provided by Trigueros lab).

Nevertheless,other $\mathrm{Au}$ nanostructures such as $\mathrm{Au}$ nanorods have also gained much attention due to their remarkable multifunctional properties especially in macrobiological system that needs the drug carriers to be half of the size of DNA molecule. For instance, Au nanorods not only can identify the attendance of tweak genes, but also assists reserchers to precisely spot the affected location of the changes. ${ }^{52}$ The assemblies of nanorods can simultaneously bind compacted genes and targeting the molceules in the acute way. The precise control of the size and structure of Au nanorods (Figure 4d) allows the efficacy in the chemotherapeutic treatment.

Presently, the controlled delivery of the active biomolecules in live cells or tissues to improve the therapeutic outcomes is one of the major targeted area in biomedicine. ${ }^{52}$ Nevertheless, the intracellular release of biomolecules in the areas of lession remains a major challenge. This due to the deficiency of physiological solubility of the biomolecules and also the low cell membrane permeability. Thus, higher dose usually preferred and this likely to cause side effects. The $\mathrm{Au}$ nanoparticles has been shown to have the potential to overcome this issues and become the ideal material for drug targeting and imaging-based detection. Due to their size smaller than the biological compounds, the active biomolecules can be loaded or attached to the surface of nanoparticles which allows delivering of those molecules directly inside the cells cytoplasm and cell organelles. Interestingly, apart from the size, this particle also exhibits high-density surface allowing high yield ligand anchorage, targeting cellular delivery, controlled intracellular release and ease the transmembrane delivery. ${ }^{52}$ Specifically, in cancer therapy, the Au nanoparticles based vehicles found to enhance the "in vivo" and "in vitro" therapeutic activity of diverse chemotherapies such as breast cancer stem cells. ${ }^{44}$ doxorubicin on human glioma cells, human melanoma cell lines..$^{39}$ temozolomide, and to promote crossing the blood barrier, thereby facilitating greater accumulation of drug in cancer cells. ${ }^{53}$

\section{Conclusion}

The serious implementation of high-throughput $\mathrm{Ag}$ and $\mathrm{Au}$ nanoparticles in the theragnostic system have devoted improved functionality result to date. While going forward, there are remains of the biggest challenge yet to be solvedin order to perceive the acceptance of these systems as first line treatment modalities. The pressing concern is to systematically deliver both $\mathrm{Ag}$ and $\mathrm{Au}$ systems in the mass balance for cancer therapies and diagnostics with efficient clearance or excretion with consideration of the subsequent accumulation of every microgram of these therapeutic drugs that are administered. It may also be better to eliminate myth and many common misapprehensions about $\mathrm{Ag}$ and $\mathrm{Au}$ nanoparticles include their high synthesis cost in amass scale and our relative lack of exposure to these systems throughout much of the $21^{\text {st }}$ century.

\section{Acknowledgment}

None

\section{Conflict of interest}

None.

\section{References}

1. Patnaik S, Gupta KC Novel Polyethylenimine-derived nanoparticles for in vivo gene delivery. Expert Opin Drug Deliv. 2013;10(2):215-228.

2. Ensign LM, Schneider C, Suk JS et al. Mucus penetrating nanoparticles: biophysical tool and method of drug and gene delivery. Adv Mater. 2012;24(28):3887-3894

3. Duceppe N, Tabrizian MAdvances in using chitosan-based nanoparticles for in vitro and in vivo drug and gene delivery. Expert Opin Drug Deliv. 2010;7(10):1191-1207.

4. Pissuwan D, Niidome T, Cortie MB The forthcoming applications of gold nanoparticles in drug and gene delivery systems. $J$ Controlled Release, 2011;149(1):65-71.

5. Ghosh P, Han G, De M et al. Gold nanoparticles in delivery applications. Adv Drug Deliv Rev. 2008;60(11):1307-1315.

6. Giljohann DA, Seferos DS, Daniel WL et al. Gold nanoparticles for biology and medicine. Angew Chem Int Ed Engl. 2010;49(19):3280-3294.

7. El Badawy A, Feldhake D, Venkatapathy R State of the Science Literature Review: Everything Nanosilver and More. US Environmental Protection Agency, Washington DC, USA. 2010 
8. Zhong W, Xing MM, Maibach HI Nano fibrous materials for wound care. Cutan Ocul Toxicol 29(3): 143-152.

9. Kim CK, Ghosh P, Pagliuca C et al. Entrapment of hydrophobic drugs in nanoparticle mono layers with efficient release into cancer cells. $J \mathrm{Am}$ Chem Soc. 2009;131(4):1360-1361.

10. Zhang XQ, Xu X, Lam R et al. Strategy for increasing drug solubility and efficacy through covalent attachment to polyvalent DNA-nanoparticle conjugates. ACS Nano. 2011;5(9):6962-6970.

11. Chen X, Schluesener HJ Nanosilver: a nano product in medical application. Toxicol Lett. 2008;176(1):1-12.

12. Takenaka S, Karg E, Roth C et al. Pulmonary and systemic distribution of inhaled ultrafine silver particles in rats. Environ Health Prospect. 2001;109(Suppl 4):547-551.

13. Y Chen, RK McCulloch, BN Gray Synthesis of albumin-dextrin sulphate microspheres possessing favourable loading and release characteristics for the anti-cancer drug doxorubicin. J Control Rel. 1994;31(1):49-54.

14. Yin WK, Feng SS Effects of particle size and surface coating on cellular uptake of polymeric nanoparticles for oral delivery of anticancer drugs. Bio materials. 2005;26(15):2713-2722.

15. Chithrani BD, Chan WCW Elucidating the mechanism of cellular uptake and removal of protein-coated gold nanoparticles of different sizes and shapes. Nano Lett. 2007;7(6):1542-1550.

16. Gao J, Gu H, Xu B Multifunctional magnetic nanoparticles: design, synthesis, and biomedical applications. Acc Chem Res. 2009;42(8):1097-1107.

17. Lai CH, Chang TC, Chuang YJ et al. Stepwise orthogonal click chemistry toward fabrication of paclitaxel/galactose functionalized fluorescent nanoparticles for HepG2 cell targeting and delivery. Bioconjug Chem. 2013;24(10):1698-1709.

18. Reidy B, Haase A, Luch A et al. Mechanisms of silver nanoparticle release, transformation and toxicity: a critical review of current knowledge and recommendations for future studies and applications. Materials. 2013;6(6):2295-2350.

19. Marradi M, Chiodo F, Garcia I, Penades S Glyco nanoparticles as multifunctional and multimodal carbohydrate systems. Chem Soc Rev. 2013;42(11):4728-4745.

20. Chiodo F, Marradi M, Calvo J et al. Glycosystems in nanotechnology: gold glyco nanoparticles as carrier for anti-HIV prodrugs. Beilstein J Org Chem. 2014;10:1339-1346.

21. Miao AJ, Schwehr KA, Xu C et al. The algal toxicity of silver engineered nanoparticles and detoxification by exopolymeric substances. Environ Pollut. 2009;157(11):3034-3041.

22. Park J, Mattessich T, Jay SM et al. Enhancement of surface ligand display on PLGA nanoparticles with amphiphilic ligand conjugates. $J$ Control Release. 2011;156(1):109-115.

23. Paciotti GF, Kingston DGI, Tamarkin L Colloidal gold nanoparticles: a novel nanoparticle platform for developing multifunctional tumortargeted drug delivery vectors. Drug Dev Res. 2006;67:47-54.

24. Longmire M, Choyke PL, Kobayashi H Clearance properties of nanosized particles and molecules as imaging agents: considerations and caveats. Future Med Nanomed. 2008;3(5):703-717.

25. Akiyama Y, Mori T, Katayama Y et al. The effects of PEG grafting level and injection dose on gold nano rod biodistribution in the tumor-bearing mice. J Control Release. 2009;139(1):81-84

26. De Jong WH, Hagens WI, Krystek P et al. Particle size-dependent organ distribution of gold nanoparticles after intravenous administration. Biomaterials. 2008;29(12):1912-1919.
27. Owens IDE, Peppas NA Opsonisation, biodistribution, and pharmacokinetics of polymeric nanoparticles. Int $J$ Pharm. 2006;307(1):93-102.

28. Nel AE, Mädler L, Velegol D et al. Understanding bio physicochemical interactions at the nano-bio interface. Nat Mater. 2009;8:543-557.

29. Melancon MP, Lu W, Zhong M et al. Targeted multifunctional goldbased nanoshells for magnetic resonance-guided laser ablation of head and neck cancer. Biomaterials. 2011;32:7600-7608.

30. Maeda $\mathrm{H}, \mathrm{Wu}$ J, Sawa $\mathrm{T}$ et al. Tumor vascular permeability and the EPR effect in macromolecular therapeutics: a review. $J$ Controlled Release. 2000;65(1-2):271-284.

31. Stevenson APZ, Blanco BD, Civit S et al. Three strategies to stabilise nearly monodispersed silver nanoparticles in aqueous solution. Nanoscale Res Lett. 2012;7(1):151-159.

32. Johnston HJ, Hutchison G, Christensen FM et al. A review of the in vivo and in vitro toxicity of silver and gold particulates: particle attributes and biological mechanisms responsible for the observed toxicity. Crit Rev Toxicol. 2010;40(4):328-346.

33. Drescher D, Orts Gil G, Laube G et al. Toxicity of amorphous silica nanoparticles on eukaryotic cell model is determined by particle agglomeration and serum protein adsorption effects. Anal Bioanal Chem. 2011;400(5):1593-1604.

34. Orts Gil G, Natte K, Drescher D et al. Characterisation of silica nanoparticles prior to in vitro studies: from primary particles to agglomerates. J Nanoparticle Res. 2011;13(4):1593-1604.

35. Lai $\mathrm{CH}$, Lin $\mathrm{CY}, \mathrm{Wu} \mathrm{HT}$ et al. Galactose encapsulated multifunctional nanoparticle for HepG2 cell internalization. Adv Funct Mater. 2010;20:3948-3958

36. Krysko DV, Vanden Berghe T, D'Herde $\mathrm{K}$ et al. Apoptosis and necrosis: detection, discrimination and phagocytosis. Methods. 2008;44(3):205-221.

37. Lee H, Park MT, Choi BH Endoplasmic reticulum stress-induced JNK activation is a critical event leading to mitochondria-mediated cell death caused by $\beta$-lapachone treatment. PLoS One. 2011;6(6):e21533.

38. Kulandaivelu B, Gothandam KM Cytotoxic Effect on Cancerous Cell Lines by Biologically Synthesized Silver Nanoparticles. Brazilian Archives of Biology and Technology. 2016;59:e16150529.

39. Kang K, Jung H, Lim JS Cell death by polyvinylpyrrolidine-coated silver nanoparticles is mediated by ROS-dependent signaling. Biomol Ther (Seoul). 2012;20(4):399-405.

40. Zhang $\mathrm{X}$ Gold nanoparticles: recent advances in the biomedical applications. Cell Biochem Biophys. 2015;72(3):771-775.

41. Thanh TD, Balamurugan J, Lee SH et al. Effective seed-assisted synthesis of gold nanoparticles anchored nitrogen-doped graphene for electrochemical detection of glucose and dopamine. Biosens Bioelect. 2016;81:259-267.

42. Nadaf NY, Kanase SS Biosynthesis of gold nanoparticles by Bacillus marisflavi and its potential in catalytic dye degradation. Arabian Journal of Chemistry. 2016

43. Bayazit MK, Yue J, Cao E et al. Controllable synthesis of gold nanoparticles in aqueous solution by microwave assisted flow chemistry. ACS Sust Chem Eng

44. Scaramuzza S, Zerbetto M, Amendola V Synthesis of gold nanoparticles in liquid environment by laser ablation with geometrically confined configurations: insights to improve size control and productivity. $J$ Phys Chem C. 2016;120(17):9453-9463.

45. Cho EJ, Holback H, Liu KC et al. Nanoparticle characterization: state of the art, challenges, and emerging technologies. Mol Pharm. 2013;10(6):2093-2110. 
46. Sun TM, Wang YC, Wang F et al. Cancer stem cell therapy using doxorubicin conjugated to gold nanoparticles via hydrazone bonds Biomaterials. 2014;35(2):836-845.

47. Halo TL, McMahon KM, Angeloni NL et al. Nano Flares for the detection, isolation, and culture of live tumor cells from human blood. Proc Nat Acad Sci. 2014;111(48):17104-17109.

48. Zandberg WF, Bakhtiari ABS, Erno Z et al. Phototherm al release of small molecules from gold nanoparticles in live cells. Nanomed. Nanotech Biol Med. 2012;8(6):908-915.

49. Hayashi K, Nakamura M, Ishimura K Near-infrared fluorescent silicacoated gold nanoparticle clusters for X-Ray computed tomography/ optical dual modal imaging of the lymphatic system. Adv Health Mat. 2013;2(5):756-763.

50. Khlebtsov N, Dykman L Biodistribution and toxicity of engineered gold nanoparticles: a review of in vitro and in vivo studies. Chem Soc Rev. 2011;40(3):1647-1671.
51. Vial S, Reis RL, Oliveira JM Recent advances using gold nanoparticles as a promising multimodal tool for tissue engineering and regenerative medicine. Cur Op Sol St Mat Sci. 2016

52. Zhang $\mathrm{X}$, Teodoro JG, Nadeau JL Intratumoral gold-doxorubicin is effective in treating melanoma in mice. Nanomedicine. 2015;11(6):1365-1375.

53. Orza A, Soritau O, Tomuleasa C et al. Reversing chemoresistance of malignant glioma stem cells using gold nanoparticles. Int J Nanomed. 2013;8:689-702. 